\title{
Improved Analysis Method for Wave-Induced Pipeline Stability on Sandy Seabed
}

\author{
Fuping $\mathrm{Gao}^{1}$; Dong-Sheng Jeng ${ }^{2}$; and Yingxiang $\mathrm{Wu}^{3}$
}

\begin{abstract}
The existing Det Norske Veritas (DNV) Recommended Practice RP E305 for pipeline on-bottom stability is mainly based on the pipe-soil interaction model reported by Wagner et al. in 1987, and the wake model reported by Lambrakos et al. in 1987, to calculate the soil resistance and the hydrodynamic forces upon pipeline, respectively. Unlike the methods in the DNV Practice, in this paper, an improved analysis method is proposed for the on-bottom stability of a submarine pipeline, which is based on the relationships between $U_{m} /(g D)^{0.5}$ and $W_{s} /\left(\gamma D^{2}\right)$ for various restraint conditions obtained by the hydrodynamic loading experiments, taking into account the coupling effects between wave, pipeline, and sandy seabed. The analysis procedure is illustrated with a detailed flow chart. A comparison is made between the submerged weights of pipeline predicted with the DNV Practice and those with the new method. The proposed analysis method may provide a helpful tool for the engineering practice of pipeline on-bottom stability design.
\end{abstract}

DOI: 10.1061/(ASCE)0733-947X(2006)132:7(590)

CE Database subject headings: Submarine pipelines; Offshore pipelines; Analytical techniques; Sea floors; Wave forces.

\section{Introduction}

During recent decades, offshore pipelines have become an efficient means of transport for oil and gas. When pipelines are installed upon seabed and subjected to wave loading, there exists a complex interaction between waves, pipelines, and seabed. To avoid the occurrence of lateral instability of a pipeline, the pipeline has to be given a heavy weight of concrete coating or alternatively be anchored/trenched. Both methodologies are expensive and complicated from the aspects of design and construction. The weight of concrete coating is a decisive factor for the accomplishment of satisfactory pipeline stability and it may prove to be the decisive factor for carrying out the installation. In the current pipeline engineering practice, however, mistakes or inconsistencies often occur in the design of the pipeline (Lawlor and Flynn 1991). The importance of a safe and economic design of submarine pipeline with respect to its on-bottom stability has been widely recognized, which increases the need for a more reliable design method.

The state-of-the-art in pipeline stability design has been changing very rapidly recently. A few investigations have addressed the problem of pipeline-seabed interaction, such as the PIPESTAB project (Brennodden et al. 1986; Wagner et al.

\footnotetext{
${ }^{1}$ Associate Professor, Institute of Mechanics, Chinese Academy of Sciences, Beijing 100080, China (corresponding author). E-mail: fpgao@imech.ac.cn

${ }^{2}$ Senior Lecturer, School of Civil Engineering, The Univ. of Sydney, Sydney, NSW 2006, Australia. E-mail: djeng@civil.usyd.edu.au

${ }^{3}$ Professor, Institute of Mechanics, Chinese Academy of Sciences, Beijing 100080, China. E-mail:yxwu@imech.ac.cn

Note. Discussion open until December 1, 2006. Separate discussions must be submitted for individual papers. To extend the closing date by one month, a written request must be filed with the ASCE Managing Editor. The manuscript for this paper was submitted for review and possible publication on May 17, 2005; approved on November 14, 2005. This paper is part of the Journal of Transportation Engineering, Vol. 132, No. 7, July 1, 2006. (CASCE, ISSN 0733-947X/2006/7-590-596/ $\$ 25.00$.
}

1987), the AGA project (Brennodden et al. 1989), and a project at the Danish Hydraulic Institute (DHI) (Palmer et al. 1988). The PIPESTAB and AGA projects have produced soil resistance models, e.g., the pipe-soil interaction model (Wagner et al. 1987) and the energy-based pipe-soil interaction model (Brennodden et al. 1989). In the previous models, which were drawn from mechanical actuator loading experiments, the complicated behavior of submarine pipelines subjected to ocean environmental loads was reflected to a certain extent. These include the pipeline embedment, which occurs as a pipeline is laid upon the seabed, and the additional settlement during oscillatory loading history. In the pipe-soil interaction model, the total soil lateral resistance to pipeline movement, $F_{H}$, was assumed to be the sum of sliding resistance component and soil passive resistance component, i.e.

$$
F_{H}=\mu\left(W_{s}-F_{L}\right)+\beta \gamma^{\prime} A_{T}
$$

where $\mu=$ sliding resistance coefficient; $W_{s}=$ pipeline submerged weight per meter, $F_{L}=$ wave induced lift force upon pipeline; $\beta=$ empirical coefficient; $\gamma^{\prime}=$ soil buoyant weight; and $A_{T}=$ half value of the contact area between pipeline and soil. From Eq. (1), the submerged weight of pipeline $\left(W_{s}\right)$ for maintaining pipeline stability can be calculated by

$$
W_{s}=\left(F_{H}-\beta \gamma^{\prime} A_{T}+\mu F_{L}\right) / \mu
$$

The above pipe-soil interaction model has been adopted in the existing Det Norske Veritas (DNV) Recommended Practice RP E305 (DNV 1988). The lateral soil resistance $\left(F_{H}\right)$ should balance the designed wave loads upon the pipeline, which can be calculated with the wake model proposed by Lambrakos et al. (1987), which takes into account of the effect of the wake generated by oscillatory flow over pipeline. In the existing DNV practice, three different methods are included, namely the dynamic analysis method, generalized stability analysis method, and simplified stability analysis method (DNV 1988). Herein, we outline these three methods: 
1. The dynamic analysis method involves a full dynamic simulation of a pipeline resting on the seabed, including modeling of soil resistance, hydrodynamic forces, boundary conditions, and pipe dynamic response. Dynamic analysis forms the reference base for the generalized analysis method. It may only be used for detailed analysis of critical areas along a pipeline, such as pipeline crossings, riser connections etc., where the details of pipeline response are required, or for reanalysis of a critical existing pipeline.

2. The generalized stability analysis is based on a set of nondimensional stability curves, which have been derived from a series of runs with a dynamic response model. This method may be used for the design of the pipeline sections where potential pipeline movement may be important. The generalized analysis method includes a complete-stable-pipeline design criterion for the special sections of a pipeline.

3. The simplified stability analysis method is for the design of common sections of a pipeline, to which an accumulative lateral displacement is allowable. It is based on a quasistatic balance of forces acting on the pipe, but has been calibrated from the generalized stability analysis. The method generally gives pipe weights that form a conservative envelope of those obtained from the generalized stability analysis.

It is noted that, in the DNV practice, the evaluation of soil resistance to pipeline movement and that of wave loads upon pipeline are conducted separately. The pipe-soil interaction model was found to be conservative for determining the weight coating of pipeline (Verley and Reed 1989). As stated by Lawlor and Flynn (1991) and Hale et al. (1991), the underlying physical mechanism of pipeline on-bottom stability has not yet been well understood.

In the previous experiments (Brennodden et al. 1986, 1989; Wagner et al. 1987; Palmer et al. 1988), the wave-induced hydrodynamic forces upon pipeline were simulated with a mechanical actuator. In their experiments, the test pipeline was attached to the mechanical rig by a suspension system, which provided the transfer of the horizontal and vertical forces simulating the wave loads on pipeline. In the models proposed by Wagner et al. (1987) and Brennodden et al. (1989), etc., numerous empirical coefficients have no implicit physical meanings and are difficult to determine in design procedure. In reality, the wave forces act not only on pipeline but also seabed, and the seabed response to the hydrodynamic forces can affect the pipeline stability too. Therefore, precisely speaking, the wave induced on-bottom stability of the submarine pipeline involves the interaction of wave, soil, and pipe, but not pipe/soil interaction.

Unlike the aforementioned experimental methods, Gao et al. (2003) have studied intensively the pipeline on-bottom stability with a U-shaped oscillatory flow tunnel, as shown in Fig. 1. The U-shaped oscillatory flow tunnel is made of transparent plexiglass with section area of $0.2 \mathrm{~m}^{2} \times 0.2 \mathrm{~m}^{2}$. By a butterfly valve periodically opening and closing at the top of a limb of the water tunnel, the water was capable of accomplishing a simple harmonic oscillation for simulating the wave induced oscillating movement of water particles near the seabed. By regulating the valve, the effective air flux from an air blower can be changed. Thus, the amplitude can be varied continuously from 5 to $200 \mathrm{~mm}$. The lower part of the water tunnel constitutes the test section with a soil box filled with sand regarded as sandy seabed. The test pipe was directly laid upon the surface of sand, as shown in Fig. 1.

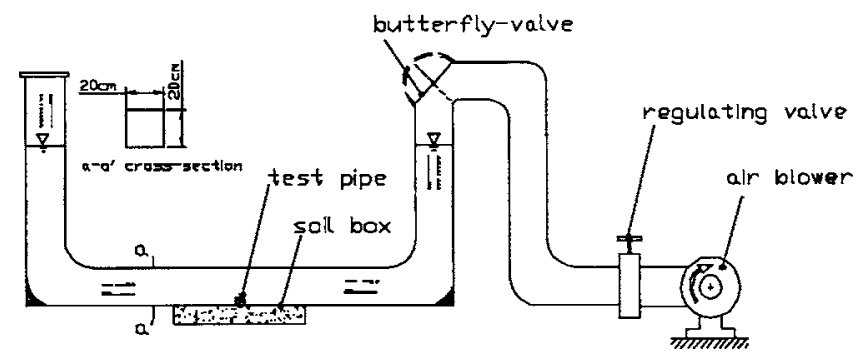

Fig. 1. U-shaped oscillatory flow tunnel for pipeline stability investigation (reprinted from Gao et al. 2003 with permission from Elsevier)

It has been widely realized that, even small change in the thickness of concrete coating may pose significant impact on the entire pipeline project (Allen et al. 1989). As such, a more reasonable analysis method for pipeline on-bottom stability is highly desired.

The objective of the paper is to propose an improved analysis method for pipeline on-bottom stability from the aspect of wavepipe-soil interaction. A comparison will be made between the physical phenomena of pipe losing stability in the pipe-soil interaction tests and those in the wave-pipe-soil interaction tests. Based on the relationships for describing pipeline on-bottom stability induced by waves, the analysis procedure will also be presented. Furthermore, the submerged weights of pipeline predicted by the improved analysis method will be compared with those by the DNV practice.

\section{Physical Phenomena of Pipeline Losing On-Bottom Stability}

In the PIPESTAB, AGA, and DHI pipe-soil interaction tests, the instability process of pipeline was either displacement controlled or force controlled. In their experiments, when losing lateral stability, pipe was pushing the soils nearby back and forth and sand scouring was not involved (Allen et al. 1989). Both the PIPESTAB and AGA pipe-soil interaction tests have generally showed that any loading history causing additional pipeline penetration would result in an increase of lateral resistance. These results can be explained by the importance of pipe penetration into the soil, mounding in front of the pipe, and the associated lateral earth passive pressure. With the increase of the sand density, the effect of cyclic preloading on the ultimate soil resistance became less, due to the reduced initial penetration in the dense sand and reduced penetration increase with cyclic loading on the dense sand compared with those for loose sand (Wagner et al. 1987). However, in oscillatory flow conditions, the traditional static stability of pipe-soil interaction is not necessarily valid. This is particularly true in the weak bottom soils typical of the upper layer of some marine sediments (Lammert and Hale 1989).

In the wave-pipe-soil interaction experiments with U-shaped oscillatory tunnel (see Fig. 1), a constant value of the increase of oscillatory flow amplitude per second, i.e., $A_{0}=0.09 \times 10^{-3} \mathrm{~m} / \mathrm{s}$, was adopted for exploration of the mechanism of pipeline instability induced by rapidly increasing storm waves. With the increase of oscillatory flow amplitude, three characteristic times are experienced during pipe losing on-bottom stability (see Fig. 2): (a) onset of scour: local scour is triggered when water particle velocity around pipe is large enough, thereafter sand ripples will 


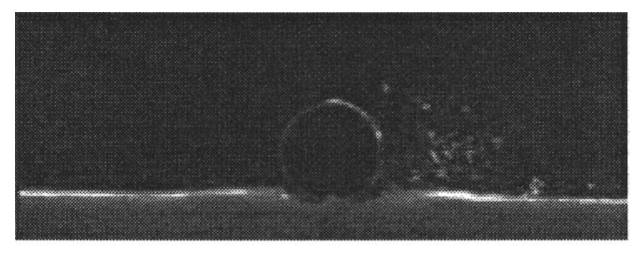

(a)

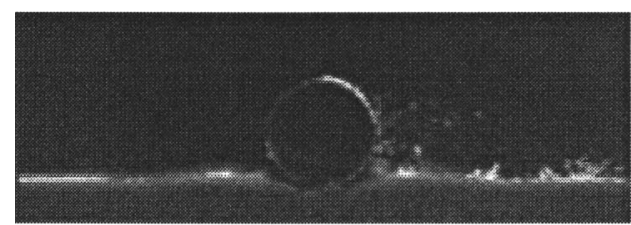

(b)

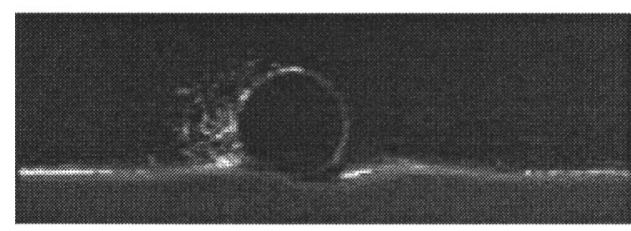

(c)

Fig. 2. Phenomena of pipeline instability in wave-pipe-soil interaction tests: (a) onset of sand scour; (b) pipeline rocks; and (c) pipeline breakouts

be gradually formed in the vicinity of the pipe; (b) pipe rocking: the pipe rocks slightly periodically at its original location with approximately same frequency of oscillatory flow; and (c) pipe breakout: the pipe suddenly moves away from its original location, or breakout takes place, after a period of slight rocking. The above process of pipeline's losing on-bottom stability has been further verified in the wave flume tests by Teh et al. (2003).

A comparison between the physical phenomena of pipe losing stability in the pipe-soil interaction tests and those in the wavepipe-soil interaction tests shows that an additional penetration of pipe into soil bed under cyclical preloadings has been found in both experiments, which increases the ultimate lateral resistance. In the wave-pipe-soil interaction tests, sand scour around the test pipe was detected and the sediment transportation was found to have an influence on pipe on-bottom stability. This, however, could not be simulated in the previous pipe-soil interaction tests. The local scour around marine structures has been recently summarized by Sumer et al. (2001) and Sumer and Fredsoe (2002).

\section{New Criteria for Pipeline On-Bottom Instability}

Dimensional analysis has indicated that the critical pipeline submerged weight $\left(W_{s} / \gamma^{\prime} D^{2}\right)$ to keep the pipeline laterally stable is mainly relative to the following parameters:

$$
\begin{aligned}
W_{s} / \gamma^{\prime} D^{2}= & f\left[U_{m} /(g D)^{0.5}, U_{m} T / D,\right. \\
& \left.U_{m} D / \nu, t / T, A_{0} / U_{m}, \rho_{\mathrm{sat}} / \rho_{w}, D / d_{s}, D_{r}, \kappa, \ldots \ldots\right]
\end{aligned}
$$

where $W_{s}=$ pipeline submerged weight per meter; $\gamma^{\prime}=\left(\rho_{\text {sat }}-\rho_{w}\right) g=$ buoyant unit weight of soil; $D=$ outer diameter

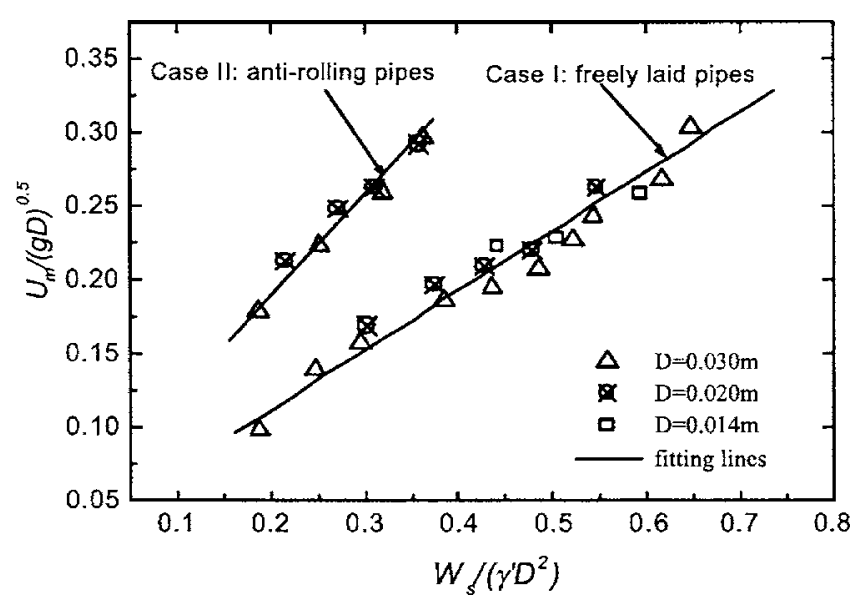

Fig. 3. $U_{m} /(g D)^{0.5}$ and $W_{s} /\left(\gamma^{\prime} D^{2}\right)$ correlation for pipeline on-bottom stability (medium sand: $D_{r}=0.37, \gamma^{\prime}=9.0 \mathrm{kN} / \mathrm{m}^{3}$ )

of pipeline; $U_{m} /(g D)^{0.5}=$ Froude number $(\mathrm{F})$, whose physical meaning is the ratio of inertia force to gravitational force; $U_{m}=$ maximum value of the velocity of water particles at seabed; $g=$ gravitational acceleration; $U_{m} T / D=$ Keulegan-Carpenter $(\mathrm{KC})$ number, which controls the generation and development of vortex around pipeline; $T=$ wave period; $U_{m} D / \nu=$ Reynolds number $(\mathrm{R})$, whose physical meaning is the ratio of inertia force to viscous force; $v=$ kinematic viscosity of fluid; $t / T=$ time of oscillatory flow acting on pipeline; $t=$ loading time; $\dot{A}_{0} / U_{m}=$ ratio of the increase of oscillatory flow amplitude per second $\left(\dot{A}_{0}\right)$ to the maximum of water particle velocity; $\rho_{\text {sat }} / \rho_{w}=$ specific gravity of saturated sand, i.e., the ratio of the density of saturated sand $\left(\rho_{\text {sat }}\right)$ to that of pore water $\left(\rho_{w}\right) ; D / d_{s}=$ ratio of pipe diameter $(D)$ to particle diameter of sand $\left(d_{s}\right) ; D_{r}==$ relative density of sand; and $\kappa=$ relative roughness of pipeline (Gao et al. 2003).

Since both $F$ and $R$ could not be satisfied concurrently in the wave experiments, it is reasonable to employ the Froude scaling process and allowances were made for variation in Reynolds number (Chakrabarti 1994). In the experiments, $R$ range is on the order of $10^{3}-10^{4}$, which is approximately one order less than that in the field. Based on the experimental results, the criteria for the stability of smooth pipelines $(\kappa \approx 0)$, on a medium-dense sand $\left(D_{r}=0.37\right)$ for two kinds of constraints, i.e., the pipe is free at its ends (Case I) and the pipe is constrained against rolling (Case II), have been established, respectively (see Fig. 3)

$$
\begin{array}{ll}
U_{m} /(g D)^{0.5}=0.042+0.38 W_{s} /\left(\gamma^{\prime} D^{2}\right) & \text { for Case I } \\
U_{m} /(g D)^{0.5}=0.069+0.62 W_{s} /\left(\gamma^{\prime} D^{2}\right) & \text { for Case II }
\end{array}
$$

The above empirical relationships are based on dimensional analysis and fitted to laboratory data. In the equations for describing pipeline on-bottom stability, the parameters for wave, pipe, and sand are coupled. The improved empirical model captures the main influential factors for on-bottom stability of pipeline laid upon sandy seabed and provides a better insight into the physical mechanics of pipeline on-bottom instability.

Scale effects should be examined when the results of smallscale wave-pipe-soil interaction tests are extrapolated to a reallife situation, where the commonly used pipe diameter is about $0.3-1.0 \mathrm{~m}$. The scale effects were investigated initially by running three scales of test pipe diameter, i.e., $D=0.014,0.020$, and $0.030 \mathrm{~m}$, respectively (see Fig. 3). Fig. 3 indicates the scale effects are not obvious when using Froude number for 


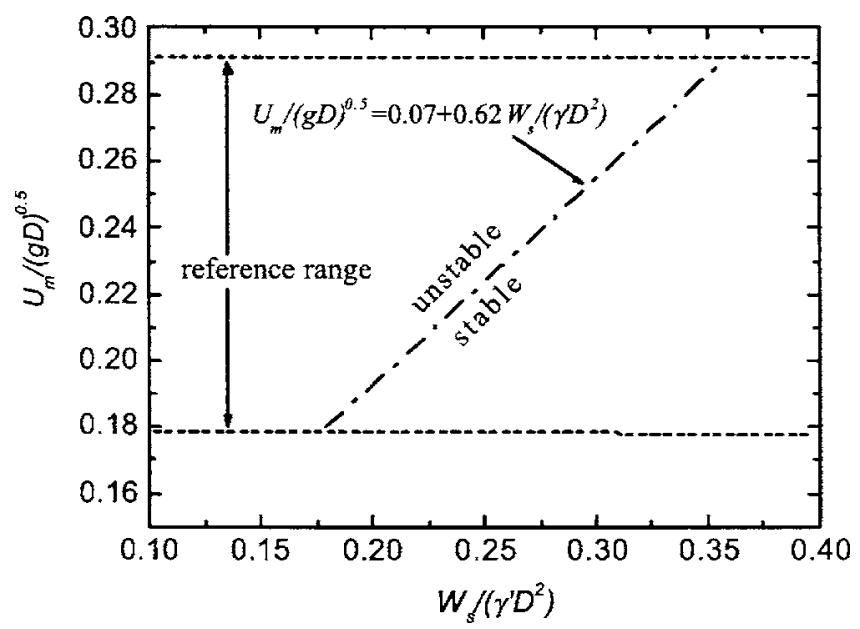

(a)

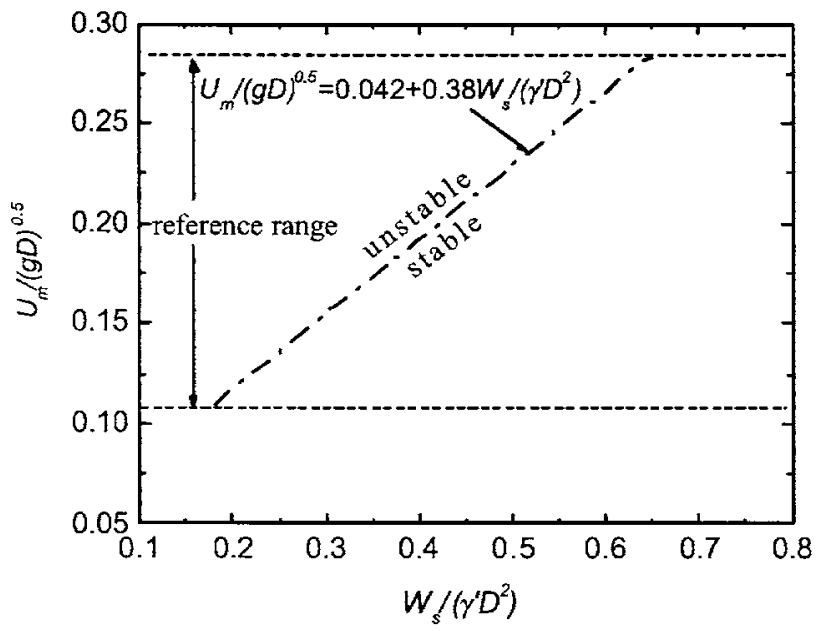

(b)

Fig. 4. Criteria for pipeline on-bottom stability on sandy medium seabed: (a) common sections of pipeline; (b) special sections of pipeline

data reduction. However, more large-scale experiments and field observations are desired to further verify the above empirical relationships. It is noted that in the experiments, $\mathrm{KC}$ range is about $5-20$, and $F$ range about $0.1-0.3$. The seabed properties and loading history would also have influence on the pipeline stability. Thus for various conditions of waves and seabed, some modification should be given to the above wave-pipe-soil interaction relationships for pipeline on-bottom stability.

According to the DNV practice (DNV 1988), for a longdistance laid pipeline, the demands for on-bottom stability are different for various sections of the pipeline. Generally speaking, no lateral displacement during extreme environmental conditions is allowed at the special locations, including the section close to a platform, normally taken as $500 \mathrm{~m}$, and some points on pipeline such as valve connections, pipeline crossing, Y- or T-connections, expansion loops, etc. Certain lateral displacement is acceptable at the common locations, i.e., the section located more than a certain

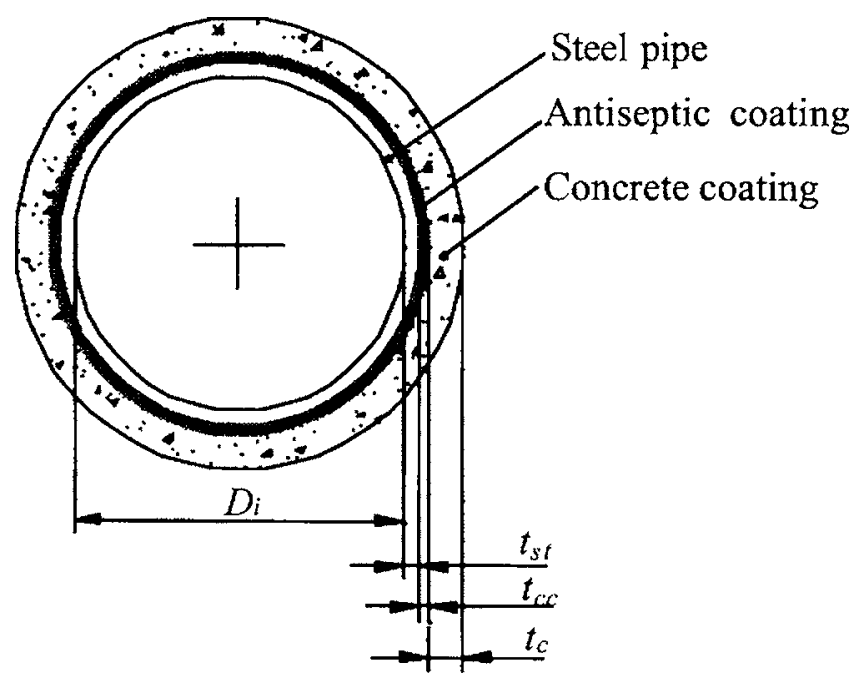

Fig. 5. Sketch of submarine pipeline wall

distance away from the platform, normally taken as $500 \mathrm{~m}$. As for the special sections of pipeline, they should remain stable even without the constraint effect from the ends. However, as for the common sections of pipeline, the demands for their stability are less rigorous, i.e., the constraint effect from the ends can be taken into account for their lateral stability. Thus, the obtained relationships in Fig. 3 may serve as the criteria for on-bottom stability of pipeline on sandy seabed. The critical line for Case I can be used for evaluating the on-bottom stability of pipeline at special locations, and the one for Case II can be used for evaluating the on-bottom stability of pipeline at common locations, as shown in Fig. 4. The critical lines in the figure are based on the medium sand test results, thus they should be modified when the physical parameters of seabed are changed. The new criteria for pipeline on-bottom stability is characterized by

- Flat seabed with homogeneous sandy soil conditions along the entire length of the pipeline;

- Waves propagating perpendicularly to the pipeline axis; and

- Materials of pipeline assumed to be rigid compared with soils.

\section{Procedure for Analysis of Wave-Induced Pipeline On-Bottom Stability}

The aim of pipeline design with respect to the on-bottom stability is to determine the steel pipeline thickness and the weight of the concrete coating or the thickness of concrete coating, so that the submerged weight of the pipeline is sufficient to meet the required stability criteria.

A typical sketch of submarine pipeline wall is illustrated in Fig. 5, in which $D_{i}$ is the inner diameter of pipeline; $t_{\mathrm{st}}, t_{\mathrm{ac}}$, and $t_{C}$ are the thickness of steel pipeline, antiseptic coating and concrete coating, respectively; $\rho_{\mathrm{st}}, \rho_{\mathrm{ac}}, \rho_{i}, \rho_{c}$, and $\rho_{w}$ are the mass density of steel, antiseptic coating, transported materials (e.g. oil), concrete coating and water. In engineering design practice, $D_{i}, t_{\mathrm{st}}, t_{\mathrm{ac}}$, $\rho_{\mathrm{st}}, \rho_{\mathrm{ac}}, \rho_{i}, \rho_{c}$, and $\rho_{w}$ are normally given first. The decisive parameter for pipeline on-bottom stability is the thickness of concrete coating $\left(t_{c}\right)$.

Based on the criteria for pipeline on-bottom stability given in the former section, an improved analysis procedure is suggested, as depicted in Fig. 6. In the steps shown in Fig. 6, for specific 


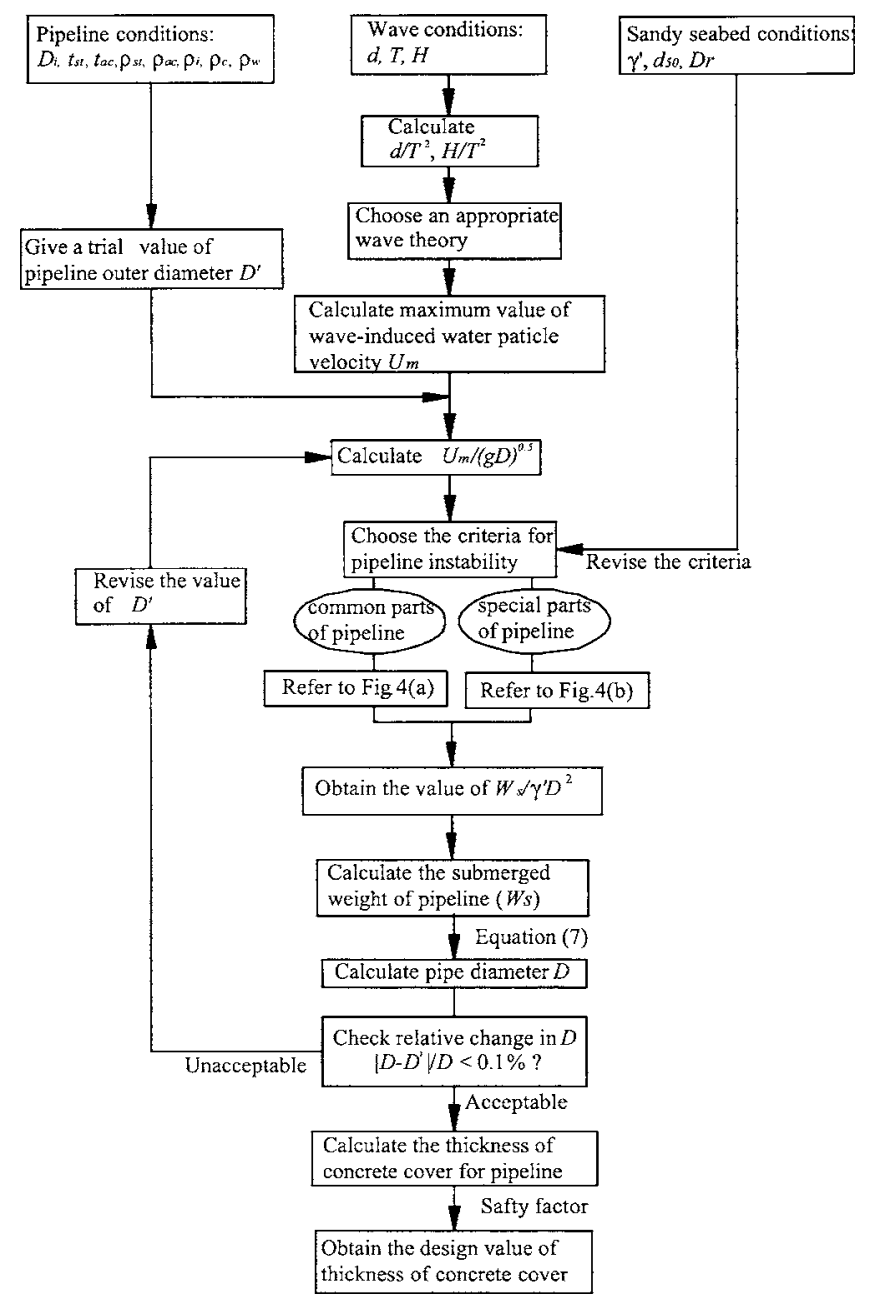

Fig. 6. Analysis procedure for pipeline on-bottom stability induced by waves

values of wave height $(H)$, wave period $(T)$, and water depth $(d)$, the maximum values of wave-induced particle velocity near seabed bottom $\left(U_{m}\right)$ can be obtained based on an appropriate wave theory. When the values of $d / T^{2}$ and $H / T^{2}$ are given, the wave theory can be chosen according to the range of suitability for wave theories as suggested by Le Mehaute (1976). In Stokes' second-order wave theory, the expression for the maximum particle velocity $U_{m}$ induced by waves is

$$
U_{m}=\frac{\pi H}{T} \frac{\operatorname{ch}[k(z+d)]}{\operatorname{sh}(k d)}+\frac{3}{4}\left(\frac{\pi H}{T}\right)\left(\frac{\pi H}{L}\right) \frac{\operatorname{ch}[2 k(z+d)]}{\operatorname{sh}^{4}(k d)}
$$

in which $k=$ wave number $(k=2 \pi / L) ; L=$ wave length; and $d=$ water depth (Sarpkaya and Isaacson 1981). For an untrenched pipeline, the value of $U_{m}$ is often chosen as that at the middle of pipeline, i.e., $z=-d+0.5 D$. Once the trial value of pipeline outer diameter $\left(D^{\prime}\right)$ is given, the Froude number, $U_{m} /(g D)^{0.5}$, can be calculated, in which $D$ will be replaced with $D^{\prime}$. Based on the criteria for pipeline on-bottom stability as shown in Fig. 4, the corresponding values of the dimensionless pipe weight $\left(W_{s} / \gamma^{\prime} D^{2}\right)$ can be obtained for the common sections or the special sections of pipeline. The submerged weight of pipeline per meter $\left(W_{s}\right)$ can thereby be calculated. Then the calculated value of pipeline diameter $(D)$ can be obtained by the following formula:

$$
D^{2}=\frac{1}{\rho_{c}-\rho_{w}}\left[\frac{4 W_{s}}{\pi g}+D_{i}^{2}\left(\rho_{\mathrm{st}}-\rho_{i}\right)+D_{\mathrm{st}}^{2}\left(\rho_{\mathrm{ac}}-\rho_{\mathrm{st}}\right)+D_{\mathrm{ac}}^{2}\left(\rho_{c}-\rho_{\mathrm{ac}}\right)\right]
$$

in which $D_{\mathrm{st}}=$ outer diameter of steel pipe $\left(D_{\mathrm{st}}=D_{i}+2 t_{\mathrm{st}}\right)$; and $D_{\mathrm{ac}}=$ outer diameter of antiseptic coating $\left(D_{\mathrm{ac}}=D_{\mathrm{st}}+2 t_{\mathrm{ac}}\right)$. If $\left|D-D^{\prime}\right| / D$ is larger than the permitting value (e.g., $0.1 \%$ ), the trail value of pipeline outer diameter $D^{\prime}$ will be revised. The thickness of concrete coating $t_{c}$ can be calculated by

$$
t_{c}=\left(D^{\prime}-D_{i}-2 t_{\mathrm{st}}-2 t_{\mathrm{cc}}\right) / 2
$$

The design value of thickness of concrete coating $\left(t_{\mathrm{cD}}\right)$ is given as

$$
t_{\mathrm{cD}}=f_{t} \times t_{c}
$$

where $f_{t}=$ safety factor, normally taken as 1.1 (DNV 1988).

\section{Comparison with DNV Recommended Practice}

The purpose of on-bottom stability design is to determine submerged weight to keep pipe stable at given environmental parameters. To compare the design results of the pipe-soil interaction model with those of the wave-pipe-soil interaction model, a pipeline with inner diameter $D=0.36 \mathrm{~m}$ (approximately $15 \mathrm{in}$.) is set as an example. The design parameters for pipeline, seabed, and waves are listed in Table 1 . The properties of sand are chosen to be the same as those of test sand in wave-pipe-soil interaction experiments; the design wave heights are various, as shown in Table 1.

Based on the generalized analysis method and the simplified analysis method in the DNV practice (DNV 1988), the design values of submerged weight $\left(W_{s}\right)$ of pipelines at various environmental parameters can be obtained respectively, which are listed

\begin{tabular}{|c|c|}
\hline Wave period $(T)$ & $9.0 \mathrm{~s}$ \\
\hline Water depth $(d)$ & $50 \mathrm{~m}$ \\
\hline Wave length $(L)$ & $125 \mathrm{~m}$ \\
\hline Wave height $(H)$ & $1.7-3.8 \mathrm{~m}$ various \\
\hline \multicolumn{2}{|l|}{ (b) Seabed (sand) characteristics } \\
\hline Mean particle diameter $\left(d_{50}\right)$ & $0.38 \mathrm{~mm}$ \\
\hline Buoyant unit weight $(\gamma)$ & $0.9 \times 10^{3} \mathrm{~N} / \mathrm{m}^{3}$ \\
\hline Relative density $\left(D_{r}\right)$ & 0.37 \\
\hline \multicolumn{2}{|l|}{ (c) Pipeline characteristics } \\
\hline Inner diameter of pipeline $\left(D_{i}\right)$ & $0.36 \mathrm{~m}$ \\
\hline Thickness of steel pipeline wall $\left(t_{\mathrm{st}}\right)$ & $0.01 \mathrm{~m}$ \\
\hline Thickness of antiseptic coating $\left(t_{\mathrm{ac}}\right)$ & $0.005 \mathrm{~m}$ \\
\hline Density of steel pipeline wall $\left(\rho_{\mathrm{st}}\right)$ & $7.85 \times 10^{3} \mathrm{~kg} / \mathrm{m}^{3}$ \\
\hline Density of transported material, e.g., crude oil $\left(\rho_{i}\right)$ & $0.95 \times 10^{3} \mathrm{~kg} / \mathrm{m}^{3}$ \\
\hline Density of sea water $\left(\rho_{w}\right)$ & $1.03 \times 10^{3} \mathrm{~kg} / \mathrm{m}^{3}$ \\
\hline Density of concrete coating $\left(\rho_{c}\right)$ & $2.40 \times 10^{3} \mathrm{~kg} / \mathrm{m}^{3}$ \\
\hline
\end{tabular}
in Table 2. Once $W_{s}$ is obtained, the dimensionless submerged

Table 1. Example of Design Parameters for Wave, Seabed, and Pipeline (a) Wave characteristics 
Table 2. Design Values of Submerged Weight of Pipelines Based on DNV Practice at Various Environmental Parameters Given in Table 1

\begin{tabular}{lccc}
\hline & & \multicolumn{2}{c}{$W_{s} /(\mathrm{kN} / \mathrm{m})$} \\
\cline { 3 - 4 }$H /(\mathrm{m})$ & $U_{m} /(\mathrm{m} / \mathrm{s})$ & $\begin{array}{c}\text { Simplified } \\
\text { analysis method }\end{array}$ & $\begin{array}{c}\text { Generalized } \\
\text { analysis method }\end{array}$ \\
\hline 1.7 & 0.25 & 0.115 & 0.288 \\
2.1 & 0.30 & 0.149 & 0.374 \\
2.4 & 0.35 & 0.187 & 0.490 \\
2.8 & 0.40 & 0.229 & 0.616 \\
3.1 & 0.45 & 0.277 & 0.748 \\
3.4 & 0.50 & 0.327 & 0.845 \\
3.8 & 0.55 & 0.385 & 1.004 \\
\hline
\end{tabular}

weight of pipeline, $W_{s} /\left(\gamma^{\prime} D^{2}\right)$, can be calculated. The values of Froude number, $U_{m} /(g D)^{0.5}$, can also be calculated. The variations of $U_{m} /(g D)^{0.5}$ with $W_{s} /\left(\gamma^{\prime} D^{2}\right)$ from the generalized analysis method, the simplified analysis method, and those from the present new analysis approach are all shown in Fig. 7.

As previously mentioned, the critical line for freely laid pipelines (Case I) can be used for evaluating the on-bottom stability of pipeline at special sections, and the one for antirolling pipelines (Case II) can be used for evaluating the on-bottom stability of pipeline at common sections. It is indicated in Fig. 7 that the critical line for the instability of anti-rolling pipeline and that for free-laid pipeline in the empirical wave-pipe-soil interaction relationships match approximately the design values based on simplified analysis method and those based on the generalized analysis method in the DNV practice, respectively. Nevertheless, with the increase of Froude number, the generalized analysis method is getting more conservative for the on-bottom stability design of the pipeline at special sections. This may be explained by the fact that the sand dune forms in the vicinity of the pipeline due to scour, and benefits the pipeline's on-bottom stability. Sand scour, as an indicator of wave-pipe-soil interaction, is one of the influential factors for pipeline stability which, however, has not been taken into account in the existing DNV practice for pipeline stability design.

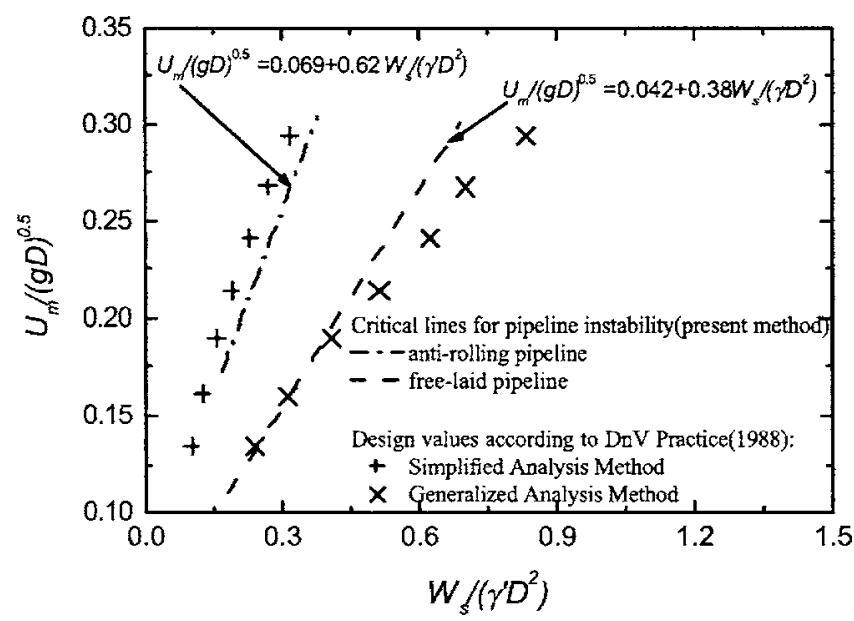

Fig. 7. Comparison between results predicted by the DNV recommended practice and by the present new analysis method

\section{Conclusions}

1. The physical phenomena of pipeline losing on-bottom stability was better simulated in the wave-pipe-soil interaction experiments, in which local scour around pipeline was detected, reflecting the intensive interaction between wave, pipe, and sand. The relationships between $U_{m} /(g D)^{0.5}$ and $W_{s} /\left(\gamma^{\prime} D^{2}\right)$ for two kinds of constraint conditions, i.e., Case I: freely laid pipes and Case II: antirolling pipes, can serve as the stability criteria for special sections and common sections of the pipeline, respectively.

2. Based on the obtained relationships between $U_{m} /(g D)^{0.5}$ and $W_{s} /\left(\gamma^{\prime} D^{2}\right)$, an improved analysis method for pipeline onbottom stability is proposed. The analysis procedure is given with a detailed illustration.

3. A comparison of submerged weights of pipeline predicted with the existing DNV Recommended Practice RP E305 and those with the new method indicates that they are generally comparable. With the increase of Froude number, the generalized method in the DNV practice becomes more conservative than the wave-pipe-soil interaction model for the on-bottom stability design of pipeline at special sections. Sand scour has some influence on pipeline stability, which however has not been considered in the existing DNV practice.

\section{Acknowledgments}

Financial support by National Natural Science Foundation of China (Project No. 50509022) and "Tenth Five-year Plan" of Chinese Academy of Sciences (Project No. KJCX2-SW-L03) is greatly appreciated. The second writer is grateful for the support from Australian Research Council Large Grant Scheme No. A00104092 (2001-2003). The writers also thank Professor Xiaoyun Gu, Professor Qun Pu, and Senior Engineer Kun Li at Institute of Mechanics, Chinese Academy of Sciences, for their guidance and assistance in the experiments.

\section{References}

Allen, D. W., Lammert, W. F., Hale, J. R., and Jacobsen, V. (1989). "Submarine pipeline on-bottom stability: Recent AGA research." Proc., 21st Annual Offshore Technology Conf., Paper OTC 6055, 121-132.

Brennodden, H., Lieng, J. T., Sotberg, T., and Verley, R. L. P. (1989). "An energy-based pipe-soil interaction model." Proc., 21st Annual Offshore Technology Conf., Paper OTC 6057, 147-158.

Brennodden, H., Sveggen, O., Wagner, D. A., and Murff, J. D. (1986). "Full-scale pipe-soil interaction tests." Proc., 18th Annual Offshore Technology Conf., Paper OTC 5338, 433-440.

Chakrabarti, S. K. (1994). Offshore structure modeling, World Scientific, River Edge, N.J.

Det Norske Veritas (DNV). (1988). "On-bottom stability design of submarine pipeline." Recommended Practice E305, DNV, Oslo, Norway.

Gao, F. P., Gu, X. Y., and Jeng, D. S. (2003). "Physical modeling of untrenched submarine pipeline instability." Ocean Eng., 30(10), 1283-1304.

Hale, J. R., Lammert, W. F., and Allen, D. W. (1991). "Pipeline onbottom stability calculations: Comparison of two state-of-the-art methods and pipe-soil model verification." Proc., 23rd Annual Offshore Technology Conf., Paper OTC 6761, 567-581. 
Lambrakos, K. F., Chao, J. C., Beckman, H., and Brannon, H. R. (1987). "Wake model of hydrodynamic forces on pipelines." Ocean Eng., 14, $117-136$.

Lammert, W. F., and Hale, J. R. (1989). "Dynamic response of submarine pipelines exposed to combined wave and current action." Proc., Annual Offshore Technology Conf., Paper OTC 6058, 159-170.

Lawlor, C. D. F., and Flynn, S. J. A. (1991). "Subsea pipeline stability analysis: Still a black art?" Trans. Inst. Eng. Aust. Civil Eng., 1, 1-8.

Le Mehaute, B. (1976). An introduction to hydrodynamics and water waves, Springer, Dusseldorf, Germany.

Palmer, A. C., Steenfelt, J. S., and Jacobsen, V. (1988). "Lateral resistance of marine pipelines on sand." Proc., 20th Annual Offshore Technology Conf., Paper OTC 5853, 399-408.

Sarpkaya, T., and Isaacson, M. (1981). Mechanics of wave forces of offshore structures, Van Nostrand Reinhold, London.
Sumer, B. M., and Fredsoe, J. (2002). The mechanics of scour in the marine environment, World Scientific, River Edge, N.J.

Sumer, B. M., Whitehouse, R. J. S., and Tørum, A. (2001). "Scour around coastal structures: A summary of recent research." Coastal Eng., 44, 153-190.

Teh, T. C., Palmer, A. C., and Damgaard, J. S. (2003). "Experimental study of marine pipelines on unstable and liquefied seabed." Coastal Eng., 50, 1-17.

Verley, R. L. P., and Reed, K. (1989). "Response of pipeline on various soils for realistic hydrodynamic loading." Proc., 8th Offshore Mechanics and Polar Engineering Conf., Vol. 5, 149-156.

Wagner, D. A., Murff, J. D., Brennodden, H., and Sveggen, O. (1987). "Pipe-soil interaction model." Proc., 19th Annual Offshore Technology Conf., Paper OTC 5504, 181-190. 